ANNALES

POLONICI MATHEMATICI

$82.1(2003)$

\title{
Foliations by planes and Lie group actions
}

\author{
by J. A. Álvarez López (Santiago de Compostela), \\ J. L. Arraut (São Carlos) and C. Biasi (São Carlos)
}

\begin{abstract}
Let $N$ be a closed orientable $n$-manifold, $n \geq 3$, and $K$ a compact nonempty subset. We prove that the existence of a transversally orientable codimension one foliation on $N \backslash K$ with leaves homeomorphic to $\mathbb{R}^{n-1}$, in the relative topology, implies that $K$ must be connected. If in addition one imposes some restrictions on the homology of $K$, then $N$ must be a homotopy sphere. Next we consider $C^{2}$ actions of a Lie group diffeomorphic to $\mathbb{R}^{n-1}$ on $N$ and obtain our main result: if $K$, the set of singular points of the action, is a finite non-empty subset, then $K$ contains only one point and $N$ is homeomorphic to $S^{n}$.
\end{abstract}

1. Introduction. A codimension one $C^{2}$ foliation defined on an $n$ manifold such that all leaves are diffeomorphic to $\mathbb{R}^{n-1}$ is called a foliation by planes. Two foliated manifolds $(V, \mathcal{F})$ and $\left(V^{\prime}, \mathcal{F}^{\prime}\right)$ are said to be $C^{r}$-conjugate if there exists a $C^{r}$ homeomorphism $h: V \rightarrow V^{\prime}$ that takes leaves of $\mathcal{F}$ onto leaves of $\mathcal{F}^{\prime}$. In this paper we first consider foliations by planes on a closed manifold $N$ minus a compact set $K$. The results obtained apply to the case of a singular foliation on $N$ defined by a $C^{2}$ integrable 1 -form for which all regular leaves are planes that cluster in $K$, which is the union of all singular leaves. The conclusions, listed below, suggest that very few closed manifolds admit singular foliations by planes. Next, we apply the same techniques to obtain information on the singular set of a $C^{2}$ action of a non-compact simply connected Lie group on a closed $n$-manifold $N$. It is well known that the singular set of a $C^{2}$ action of $\mathbb{R}$ on $N$ is generically a finite subset, but very little is known when the group acting is diffeomorphic to $\mathbb{R}^{n-1}$. Here we prove (see Theorem 2.9) that the singular set $K$ of a $C^{2}$ action of a Lie group $G$ diffeomorphic to $\mathbb{R}^{n-1}$ on $N$ cannot be a finite

2000 Mathematics Subject Classification: Primary 57S25; Secondary 57R25, 57R30.

Key words and phrases: foliation by planes, homotopy spheres, actions.

Research of J. A. Álvarez López partially supported by the "Ministerio de Educación y Ciencia" (Spain), grant \# BFM2001-3280.

Research of J. L. Arraut and C. Biasi partially supported by FAPESP, grant \# 00/05385-8. 
non-empty set unless $N$ is homeomorphic to $S^{n}$, and in this case $K$ contains exactly one point. What is generically the singular set of those actions is an open and difficult question.

2. Statements of the results. Given a manifold $M$ we shall denote by $M^{*}$ its one-point compactification and by $P: \widetilde{M} \rightarrow M$ its universal covering map. If $A$ is a subset of $M$, define $\widetilde{A}=P^{-1}(A)$. If $\mathcal{F}$ is a foliation of $M$, then its lift to $\widetilde{M}$ will be denoted by $\widetilde{\mathcal{F}}$.

Proposition 2.1. Let $M$ be an n-manifold foliated by planes. Then $\pi_{1}(M)$ is torsion-free.

Proposition 2.2. Let $M$ be an open connected $n$-manifold, $n \geq 3$, and $K$ a closed subset such that $\pi_{1}(M \backslash K)$ is finitely generated. If there exists a transversely orientable foliation of $M \backslash K$ by planes such that each leaf is closed, then $K^{*}$ is connected.

Corollary 2.3. Under the hypothesis of Proposition 2.2:

(i) If $K \neq \emptyset$, then no connected component of $K$ is compact. In particular, if $K$ is compact, then $K=\emptyset$.

(ii) If $\operatorname{dim}_{\text {top }} K=0$, then $K=\emptyset$.

Proposition 2.4. Let $N$ be a closed, connected and orientable $n$-manifold, $n \geq 3$, and $K$ a compact non-empty subset. If $\pi_{1}(N \backslash K)$ is finitely generated and there exists a transversely orientable foliation of $N \backslash K$ by planes such that each leaf is closed, then $K$ is connected.

Due to Proposition 2.4, there is no loss of generality if one assumes, in the next two theorems, that $K$ is connected.

Recall that a space is called a homology sphere when its homology is isomorphic to the homology of some sphere, and it is called a homotopy sphere if its homotopy groups are isomorphic to the corresponding homotopy groups of some sphere.

THEOREM 2.5. Let $N$ be a closed, connected and orientable $n$-manifold, $n \geq 3$, and $K \subset N$ a non-empty, compact and connected ANR. Assume that $H_{p}(K ; \mathbb{Z})=0$ for $0<p \leq[n / 2]$. If $\pi_{1}(N \backslash K)$ is finitely generated and there exists a transversely orientable foliation of $N \backslash K$ by planes such that each leaf is closed, then $N$ is a homology sphere.

Theorem 2.6. Let $N$ and $K$ be as in Theorem 2.5 and assume, moreover, that $H^{n-2}(K ; \mathbb{Z})=0$ and $\operatorname{dim}_{\text {top }} K \leq n-2$. Then $N$ is a homotopy sphere for $n=3$, and homeomorphic to $S^{n}$ if $n \geq 4$.

Corollary 2.7. Let $N$ be a closed, connected and orientable $n$-manifold, $n \geq 3$, and $K$ a non-empty compact subset with $\operatorname{dim}_{\text {top }} K=0$. If 
$\pi_{1}(N \backslash K)$ is finitely generated and there exists a transversely orientable foliation of $N \backslash K$ by planes such that each leaf is closed, then:

(i) $K$ contains only one point,

(ii) $N$ is homeomorphic to $S^{n}$.

THEOREM 2.8. Let $N$ be a closed, connected and orientable 3-manifold, and $K$ a circle embedded in $N$. Suppose that there exists a transversally orientable foliation of $N \backslash K$ by planes such that each leaf is closed. Then $N$ admits a Heegaard diagram of genus one, and therefore $\pi_{1}(N)$ is a cyclic group. Moreover:

(i) if $\pi_{1}(N)=0$, then $N$ is homeomorphic to $S^{3}$,

(ii) if $\pi_{1}(N)=\mathbb{Z}$, then $N$ is homeomorphic to $S^{1} \times S^{2}$.

Now, let $G$ denote a Lie group diffeomorphic to $\mathbb{R}^{n-1}$. For $n-1=2$, there are two such Lie groups: $\mathbb{R}^{2}$ and the group $A^{2}$ of orientation preserving affine transformations of $\mathbb{R}$. Given an action $A: N \times G \rightarrow N$, a point $p$ is said to be a singular point of $A$ if the orbit of $p$ has topological dimension strictly less than $n-1$. In the following propositions it will not be necessary to assume that $N$ is orientable. Also, instead of assuming that $N \backslash K$ is foliated by planes, we shall assume that on $N$ there is given a $C^{2}$ action of a Lie group diffeomorphic to $\mathbb{R}^{n-1}$.

THEOREM 2.9. Let $N$ be a closed and connected $n$-manifold, $n \geq 3$, with a $C^{2}$ action of a Lie group $G$ diffeomorphic to $\mathbb{R}^{n-1}$. Assume that the set $K$ of singular points of the action is non-empty and finite. Then:

(i) $K$ contains only one point,

(ii) $N$ is homeomorphic to $S^{n}$.

Corollary 2.10. Let $N$ be a closed and connected $n$-manifold, $n \geq 3$, with a $C^{2}$ action of a Lie group $G$ diffeomorphic to $\mathbb{R}^{n-1}$. Assume that $K$ is composed of $k$ orbits with $k \neq 0$. Then:

(i) if $N \neq S^{n}$ then at least one orbit has dimension greater than or equal to one,

(ii) if $N=S^{n}$ and $k \geq 2$, then at least one orbit has dimension greater than or equal to one.

THEOREM 2.11. Let $N$ be a closed and connected $n$-manifold, $n \geq 3$, with a $C^{2}$ action of a Lie group $G$ diffeomorphic to $\mathbb{R}^{n-1}$. Assume that the singular set $K$ of the action is a Whitney stratified set that contains at least one stratum of dimension $n-2$. If $A_{i}$ is a connected component of $K$ with $\operatorname{dim} A_{i} \leq n-3$, then the homomorphism $\pi_{1}\left(A_{i}\right) \rightarrow \pi_{1}(N)$, induced by the inclusion map $A_{i} \hookrightarrow N$, is not the zero map. 
Corollary 2.12. Let $N$ be a closed orientable 3 -manifold with a $C^{2}$ action of a Lie group $G$ diffeomorphic to $\mathbb{R}^{2}$. Assume that the singular set $K$ of the action is a Whitney stratified non-empty set. Then:

(i) if $\operatorname{dim} K=0$, then $N=S^{3}$ and $K$ contains only one point;

(ii) if $\operatorname{dim} K=1$, then $K$ does not contain isolated points.

\section{Examples}

EXAMPLE 3.1. Consider the singular foliation of $S^{2}$ whose regular leaves are the meridians and the singular ones are the poles $P_{1}$ and $P_{2}$, and form the product $S^{2} \times[0,1]$. Next, identify each $(x, 1)$ with $(\psi(x), 0)$, where $\psi$ : $S^{2} \rightarrow S^{2}$ is a rotation, fixing the poles, of angle $\alpha$ such that the numbers $\alpha$ and $2 \pi$ are linearly independent over $\mathbb{Q}$. In this way one obtains a foliation of $N=S^{2} \times S^{1}$ by planes with singular set $K=\left(\left\{P_{1}\right\} \times S^{1}\right) \cup\left(\left\{P_{2}\right\} \times S^{1}\right)$, which is not connected. Notice that here the regular leaves are not closed in $N \backslash K$, instead they are dense in $N$.

EXAMPLE 3.2. Let $\left(x_{1}, \ldots, x_{n}\right)$ be the standard coordinates of $\mathbb{R}^{n}$, and let $r^{2}=x_{1}^{2}+\ldots+x_{n}^{2}$. Then the form $d x_{n}$ defines a foliation of $\mathbb{R}^{n}=S^{n} \backslash\{\infty\}$ by closed planes, and the form $e^{-r^{2}} d x_{n}$ defines a singular foliation of $S^{n}$ by planes with $\{\infty\}$ as the only singular leaf.

EXAmple 3.3. Let $S^{n}=\left\{x \in \mathbb{R}^{n+1} \mid x_{1}^{2}+\ldots+x_{n}^{2}=1\right\}, F=(0, \ldots, 0,1)$, $\mathbb{R}^{n}=\left\{x \in \mathbb{R}^{n+1} \mid x_{n+1}=0\right\}$, and $P: S^{n} \backslash F \rightarrow \mathbb{R}^{n}$ be the projection with $F$ as focus. The vector fields $P_{*}^{-1}\left(\partial / \partial x_{j}\right), 1 \leq j \leq n-1$, defined on $S^{n} \backslash F$ extend to $C^{\infty}$ vector fields $X_{j}$ on $S^{n}$ and clearly any two of them commute. They define an action of $\mathbb{R}^{n-1}$ on $S^{n}$ where all regular orbits are planes that cluster at the stationary point $F$.

EXAMPLE 3.4. Consider the following three foliations on $S^{1} \times D^{2}$, the compact solid torus. Using $(\phi,(x, y))$ as coordinates, put $\omega_{1}=d \phi$ and $\omega_{2}=$ $q^{*}(-y d x+x d y)$, where $q: S^{1} \times D^{2} \rightarrow D^{2}$ is the projection. The leaves of the foliation $\mathcal{F}_{1}$ defined by $\omega_{1}$ are the disks $\{\phi\} \times D^{2}$. The regular leaves of the foliation $\mathcal{F}_{2}$ defined by $\omega_{2}$ are of the form $S^{1} \times\{$ ray $\}$, and the singular leaf is the central circle $K=S^{1} \times\{0\} . \mathcal{F}_{3}$ is obtained from $\mathcal{F}_{1}$ by turbulizing the disks along the central circle. Now consider a copy of the solid torus with the foliation $\mathcal{F}_{1}$ and another copy with $\mathcal{F}_{2}$, and identify their boundaries through the map that sends meridians onto parallels. One obtains a foliation of $S^{3} \backslash K$ by closed planes. If one uses $\mathcal{F}_{1}$ on one copy and $\mathcal{F}_{3}$ on the other and identify by means of the identity map of the boundary, then one obtains a foliation of $\left(S^{1} \times S^{2}\right) \backslash K$ by closed planes. 
4. Proofs of the results. In this section, we give the proof of the statements of Section 2. In [6], Palmeira studied foliations by planes on open manifolds. He proved the following theorem.

THEOREM 4.1. If $V$ is an orientable open $n$-manifold, $n \geq 3$, which has a finitely generated fundamental group and with a transversely orientable $C^{2}$ foliation $\mathcal{F}$ by closed planes, then there exists an orientable surface $\Sigma$ and an orientable one-dimensional foliation $\mathcal{F}_{0}$ of $\Sigma$ such that $(V, \mathcal{F})$ is conjugate by a diffeomorphism to $\left(\Sigma \times \mathbb{R}^{n-2}, \mathcal{F}_{0} \times \mathbb{R}^{n-2}\right)$. When $V$ is simply connected it is not necessary to assume either that $\mathcal{F}$ is transversely orientable or that the leaves are closed, and moreover $\Sigma=\mathbb{R}^{2}$ in this case.

REMARK 1. In Theorem 4.1, each connected component of $\Sigma$ can only be an open surface or a torus $S^{1} \times S^{1}$. Since the leaves of $\mathcal{F}_{0}$ are homeomorphic to $\mathbb{R}$ and closed in $\Sigma$, it follows that no connected component of $\Sigma$ is a torus. So all connected components of $\Sigma$ are open surfaces.

We start with a corollary that translates Theorem 4.1 into cohomological information.

Corollary 4.2. If $V$ and $\mathcal{F}$ are as in Theorem 4.1, then $H_{p}(V)=0$ and $H^{p}(V)=0$ for $p \geq 2$.

Proof. By Theorem 4.1, there exists an orientable surface $\Sigma$ and an orientable one dimensional foliation $\mathcal{F}_{0}$ of $\Sigma$ such that $(V, \mathcal{F})$ is conjugate by a diffeomorphism to $\left(\Sigma \times \mathbb{R}^{n-2}, \mathcal{F}_{0} \times \mathbb{R}^{n-2}\right)$. In particular, $V$ and $\Sigma$ have the same homotopy type, and thus $H_{p}(V) \cong H_{p}(\Sigma)$ for each $p$. Moreover all connected components of $\Sigma$ are open surfaces by Remark 1, and thus $H_{p}(V) \cong H_{p}(\Sigma)=0$ and $H^{p}(V) \cong H^{p}(\Sigma)=0$ for each $p \geq 2$.

Proof of Proposition 2.1. To prove that $\pi_{1}(M)$ is torsion-free, it is enough to show that its only finite subgroup is the trivial one. Let $\mathcal{F}$ denote the foliation of $M$ by planes, let $H$ be a finite subgroup of $\pi_{1}(M)$, and let $k$ denote the number of elements of $H$. Let $\widetilde{M} \rightarrow M$ be the universal covering map, and let $\widehat{M} \rightarrow M$ be the covering map associated to $H$, i.e., $\pi_{1}(\widehat{M})=H$. Let $\widetilde{\mathcal{F}}$ and $\widehat{\mathcal{F}}$ be the foliations of $\widetilde{M}$ and of $\widehat{M}$ induced by $\mathcal{F}$; both are foliations by planes. We have $\widetilde{M}=\mathbb{R}^{n}$ by the last part of Theorem 4.1 . Then the Euler characteristics of $\widetilde{M}$ and $\widehat{M}$ satisfy

$$
1=\chi\left(\mathbb{R}^{n}\right)=\chi(\widetilde{M})=k \cdot \chi(\widehat{M}), \quad \chi(\widehat{M}) \in \mathbb{Z},
$$

yielding $k=1$ and $H=0$.

Proof of Proposition 2.2. Consider the exact sequence of Čech cohomology groups with coefficients in $\mathbb{Z}_{2}$ :

$$
H^{0}\left(M^{*}, K^{*}\right) \rightarrow H^{0}\left(M^{*}\right) \rightarrow H^{0}\left(K^{*}\right) \rightarrow H^{1}\left(M^{*}, K^{*}\right) \rightarrow \ldots
$$


The pair $\left(M^{*}, K^{*}\right)$ is a relative manifold, i.e., $M^{*}$ is Hausdorff and compact, $K^{*} \subset M^{*}$ is closed and $M^{*} \backslash K^{*}$ is an $n$-manifold. Then we have the isomorphisms

$$
H^{p}\left(M^{*}, K^{*}\right) \cong H_{n-p}\left(M^{*} \backslash K^{*}\right)=H_{n-p}(M \backslash K)
$$

for $0 \leq p \leq n$, given by the Alexander-Cech duality. If we replace $H^{0}\left(M^{*}, K^{*}\right)$ by $H_{n}(M \backslash K)$ and $H^{1}\left(M^{*}, K^{*}\right)$ by $H_{n-1}(M \backslash K)$ in the exact sequence (1), and use Corollary 4.2 with $V=M \backslash K$, we get the short exact sequence

$$
0 \rightarrow \mathbb{Z}_{2} \rightarrow H^{0}(K) \rightarrow 0 .
$$

Thus $H^{0}(K)=\mathbb{Z}_{2}$, and consequently $K^{*}$ is connected.

Proof of Proposition 2.4. Consider the exact sequence of singular homology groups with coefficients in $\mathbb{Z}$ :

$$
\ldots \rightarrow H_{p+1}(N, N \backslash K) \rightarrow H_{p}(N \backslash K) \rightarrow H_{p}(N) \rightarrow H_{p}(N, N \backslash K) \rightarrow \ldots
$$

and the isomorphisms

$$
H_{p}(N, N \backslash K) \cong H^{n-p}(K)
$$

for $0 \leq p \leq n$, given by Alexander-Poincaré duality. We are using Čech cohomology for $K$. Then, by replacing $H_{n}(N, N \backslash K)$ by $H^{0}(K)$ in the exact sequence (2), and by using Corollary 4.2 with $V=N \backslash K$, one obtains the short exact sequence

$$
0 \rightarrow \mathbb{Z} \rightarrow H^{0}(K) \rightarrow 0 .
$$

Thus $H^{0}(K) \cong \mathbb{Z}$, and consequently $K$ is connected.

Proof of Theorem 2.5. Consider the singular homology exact sequence of the pair $(N, K)$ with coefficients in $\mathbb{Z}$ :

$$
\ldots \rightarrow H_{p}(K) \rightarrow H_{p}(N) \rightarrow H_{p}(N, K) \rightarrow H_{p-1}(K) \rightarrow \ldots,
$$

and also the homology and cohomology groups of $N \backslash K$. Since $K$ is an ANR (absolute neighbourhood retract) we have, by duality, the isomorphisms

$$
H_{p}(N, K) \cong H^{n-p}(N \backslash K)
$$

for $p \geq 0$. Therefore the exact sequence (3) can be written as

$$
\ldots \rightarrow H_{p}(K) \rightarrow H_{p}(N) \rightarrow H^{n-p}(N \backslash K) \rightarrow H_{p-1}(K) \rightarrow \ldots
$$

By assumption, we have $H_{p}(K)=0$ for $1 \leq p \leq[n / 2]$. Moreover $H^{n-p}(N \backslash K)=0$ for $1 \leq p \leq[n / 2]$ by Corollary 4.2 since $n-p \geq 2$. From (4) we obtain $H_{p}(N)=0$ for $1 \leq p \leq[n / 2]$, yielding $H^{n-p}(N)=0$ by Poincaré duality. Hence $H_{p}(N)=0$ for $1 \leq p \leq n-1$ since we can write

$$
H_{p}(N)=F_{p} \oplus T_{p}, \quad H^{p}(N)=F_{p} \oplus T_{p-1},
$$

where $F$ denotes the free part and $T$ the torsion part (see e.g. [3, p. 136]). 
Proof of Theorem 2.6. We already know, by Theorem 2.5, that $N$ is a homology sphere. To see that $N$ is a homotopy sphere, it is enough to prove that $\pi_{1}(N)=0$. This simplification can be proved as follows. Since $N$ is a homology sphere and a closed connected oriented $n$-manifold, its homology is isomorphic to the homology of $S^{n}$. Hence $\pi_{i}(N)=0$ for $0<i<n$ and $\pi_{n}(N) \cong \mathbb{Z}$ by the Hurewicz isomorphism theorem [8, pp. 397-398]. Then, for any map $f: S^{n} \rightarrow N$ representing a generator of $\pi_{n}(N)$, the induced map $f_{*}: H_{n}\left(S^{n}\right) \rightarrow H_{n}(N)$ is an isomorphism, and thus $f_{*}: H_{i}\left(S^{n}\right) \rightarrow$ $H_{i}(N)$ is an isomorphism for all $i$. Therefore $\pi_{i}(f): \pi_{i}\left(S^{n}\right) \rightarrow \pi_{i}(N)$ is an isomorphism for all $i$ by the Whitehead theorem [8, p. 399], which is a consequence of the Hurewicz isomorphism theorem.

From the exact sequence (2), using $H^{n-2}(K)=0$ and $H^{1}(N)=0$, one obtains $H^{1}(N \backslash K)=H^{1}(\Sigma)=0$ with the notation of Theorem 4.1 for $V=N \backslash K$. So $\Sigma$ is diffeomorphic to $\mathbb{R}^{2}$, and thus $N \backslash K$ is diffeomorphic to $\mathbb{R}^{n}$, yielding $\pi_{1}(N \backslash K)=0$. Finally, since $\operatorname{dim}_{\text {top }} K \leq n-2$ and $N$ is a Cantor manifold [5, p. 93], it follows that the map $\pi_{1}(N \backslash K) \rightarrow \pi_{1}(N)$ induced by the inclusion is surjective, and consequently $\pi_{1}(N)=0$ as desired. The fact that $N$ is homeomorphic to $S^{n}$ for $n \geq 4$ follows from the celebrated theorems of Freedman [1] (for $n=4$ ) and Smale [7] (for $n \geq 5)$.

Proof of Corollary 2.7. $K$ is connected by Proposition 2.4. So $K$ reduces to a point since $\operatorname{dim}_{\text {top }} K=0$. By Theorem 2.6, $N$ is a homotopy sphere, and therefore homeomorphic to $S^{n}$ for $n \geq 4$. For $n=3$, the fact that $K$ is a point and $N \backslash K$ is homeomorphic to $\mathbb{R}^{3}$ implies that $N$ is homeomorphic to $S^{3}$ as well.

Proof of Theorem 2.8. Let $T(K)$ be an open tubular neighbourhood of $K$ diffeomorphic to the solid torus $S^{1} \times D^{2}$, and let $V=N \backslash \overline{T(K)}$. Observe that $N \backslash K$ is homotopic to $N \backslash T(K)$, which is a compact manifold with boundary, and thus $\pi_{1}(N \backslash K)$ is finitely generated. Since $V$ is diffeomorphic to $N \backslash K$, we see that $V$ satisfies the assumptions of Theorem 4.1. Therefore $V$ is diffeomorphic to $\Sigma \times \mathbb{R}$ for some connected orientable surface $\Sigma$, which is open by Remark 1 . We have $H_{p}(V)=H_{p}(\Sigma)=0$ for $p \geq 2$ by Corollary 4.2. From the formula $\chi(N)=\chi(V)-\chi(\overline{T(K)})$, which relates the Euler characteristics, the Betti numbers of $\Sigma$ satisfy

$$
0=\beta_{0}(\Sigma)-\beta_{1}(\Sigma)=1-\beta_{1}(\Sigma),
$$

i.e., $\beta_{1}(\Sigma)=1$. Since the first Betti number is a complete invariant for connected orientable surfaces when it is finite, it follows that $\Sigma$ is homeomorphic to $S^{1} \times(0,1)$, and consequently $V$ is homeomorphic to $S^{1} \times D^{2}$. Thus $N$ is obtained by pasting two copies of $S^{1} \times D^{2}$, which means that $N$ admits a Heegaard splitting of genus one. 
Proof of Theorem 2.9. Let $\tilde{N}$ be the universal covering space of $N$. Since $G$ is simply connected, the $C^{2}$ action of $G$ on $N$ lifts to a $C^{2}$ action of $G$ on $\widetilde{N}$. The singular points of this action on $\widetilde{N}$ are those over points in $K$, and they form a discrete subset $\widetilde{K} \subset \widetilde{N}$. Moreover this action on $\widetilde{N}$ defines a foliation $\widetilde{\mathcal{F}}$ on $\widetilde{N} \backslash \widetilde{K}$, which is the lift of $\mathcal{F}$.

For any leaf $L$ of $\widetilde{\mathcal{F}}$, consider the homomorphism $\pi_{1}(j): \pi_{1}(L) \rightarrow$ $\pi_{1}(\widetilde{N} \backslash \widetilde{K})$ induced by the inclusion map $j: L \hookrightarrow \widetilde{N} \backslash \widetilde{K}$. On the one hand, we know that $\pi_{1}(\widetilde{N} \backslash \widetilde{K}) \cong \pi_{1}(\widetilde{N})=0$. On the other hand, $\pi_{1}(j)$ is injective. In fact, if $\pi_{1}(j)$ were not injective, then $\widetilde{\mathcal{F}}$ would have a vanishing cycle by a theorem of Novikov [2, p. 265]; but a foliation defined by a locally free action of a Lie group has no vanishing cycles [2, p. 270]. Therefore $\pi_{1}(L)=0$, and thus the isotropy group at any point of $L$ is trivial. So $L$ is diffeomorphic to $G$, and thus to $\mathbb{R}^{n-1}$; i.e., $\widetilde{\mathcal{F}}$ is a foliation by planes. Moreover the leaves of $\widetilde{\mathcal{F}}$ are closed in $\widetilde{N} \backslash \widetilde{K}[2$, p. 270]. Then, by Theorem 4.1, $\widetilde{N} \backslash \widetilde{K}$ is diffeomorphic to $\mathbb{R}^{n}$, which has only one end. But any point of $\widetilde{K}$ can be considered as an end of $\widetilde{N} \backslash \widetilde{K}$ because $\widetilde{K}$ is discrete in $\widetilde{N}$, which shows that $\widetilde{K}$ contains only one point. Hence $K$ contains only one point as well, and $\widetilde{N}$ is a one-fold covering of $N$, i.e., $\widetilde{N}=N$. So $N$ is the one-point compactification of $\mathbb{R}^{n}$, and thus homeomorphic to $S^{n}$.

Proof of Theorem 2.11. Recall that the dimension of a Whitney stratified set $X$ is the maximum of the dimensions of its strata, which equals its topological dimension because $X$ is triangulable according to a result of M. Goresky.

Consider the decomposition of $K$ into its connected components, which are also Whitney stratified sets:

$$
K=A_{1} \cup \ldots \cup A_{\alpha} \cup B_{1} \cup \ldots \cup B_{\beta},
$$

where $\operatorname{dim} A_{i} \leq n-3, i=1, \ldots, \alpha$, and $\operatorname{dim} B_{j}=n-2, j=1, \ldots, \beta$. Let $A=A_{1} \cup \ldots \cup A_{\alpha}$ and $B=B_{1} \cup \ldots \cup B_{\beta}$. By assumption $B \neq \emptyset$, thus $M=N \backslash B$ is an open manifold because $B$ is compact. Since $G$ is simply connected, the $C^{2}$ action of $G$ on $M$ can be lifted to a $C^{2}$ action of $G$ on $\widetilde{M}$. The singular set $\widetilde{A}$ of this action is the inverse image of $A$. Consider the commutative diagram induced by inclusions and projections

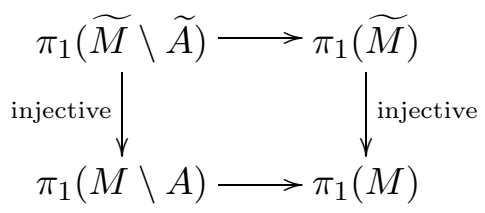

Note that $M$ is homotopic to a compact manifold with boundary (the complement in $N$ of an appropriate open neighbourhood of $B$ ); so $\pi_{1}(M)$ is finitely generated. It follows that the map $\pi_{1}(M \backslash A) \rightarrow \pi_{1}(M)$ is an iso- 
morphism since $\operatorname{dim} A \leq n-3$ ( $A$ is a finite union of manifolds of dimension $\leq n-3)$. Therefore $\pi_{1}(\widetilde{M} \backslash \widetilde{A})=0$. Let $\mathcal{F}$ be the foliation of $M \backslash A$ defined by the orbits of the action. One deduces, as in the proof of Theorem 2.9, that $\widetilde{\mathcal{F}}$ is transversely orientable with closed leaves. Then $\widetilde{M} \backslash \widetilde{A}$ is diffeomorphic to $\mathbb{R}^{n}$ by Theorem 4.1 , and $(\widetilde{A})^{*}$ is connected by Proposition 2.2 . It follows that each $\widetilde{A}_{i}$ is non-compact, and thus the projection $\widetilde{A}_{i} \rightarrow A_{i}$ is a non-trivial covering map. So $\pi_{1}\left(A_{i}\right) \rightarrow \pi_{1}(N)$ is not the zero map because its image can be canonically identified with the group of deck transformations of $\widetilde{A}_{i} \rightarrow A_{i}$.

\section{References}

[1] M. H. Freedman, The topology of four-dimensional manifolds, J. Differential Geom. 17 (1982), 357-453.

[2] C. Godbillon, Feuilletages. Études Géométriques, Progr. Math. 98, Birkhäuser, 1991.

[3] M. Greenberg, Lectures on Algebraic Topology, Benjamin, New York, 1967.

[4] A. Haefliger, Sur les feuilletages des variétés de dimension $n$ par des feuilles fermées de dimension $n-1$, in: Colloque de Topologie de Strasbourg, 1955, 8 pp.

[5] W. Hurewicz and H. Wallman, Dimension Theory, Princeton Univ. Press, Princeton, 1948.

[6] C. F. B. Palmeira, Open manifolds foliated by planes, Ann. of Math. 107 (1978), 109-131.

[7] S. Smale, Generalized Poincaré's conjecture in dimensions greater than four, ibid. 74 (1961), 391-406.

[8] E. H. Spanier, Algebraic Topology, McGraw-Hill, New York, 1966.

Departamento de Xeometría e Topoloxía Facultade de Matemáticas

Universidade de Santiago de Compostela 15706 Santiago de Compostela, Spain

E-mail: jalvarez@usc.es
Departamento de Matemática Instituto de Matemática e Computação Universidade de São Paulo Campus de São Carlos Caixa Postal 668 13560-970 São Carlos SP, Brasil

E-mail: arraut@icmc.usp.br biasi@icmc.usp.br 